\title{
Interaction of Eddies and Mean Zonal Flow on Jupiter as Inferred From Voyager 1 and 2 Images
}

\author{
Andrew P. Ingersoll, ${ }^{1}$ Reta F. Beebe, ${ }^{2}$ Jim L. Mitchell, ${ }^{3}$ Glenn W. Garneau, ${ }^{4}$ Gary M. Yagi, ${ }^{4}$ \\ AND JAN-PETER MÓLLER ${ }^{5}$
}

\begin{abstract}
Voyager 1 and 2 narrow-angle frames were used to obtain displacements of features at resolutions of $130 \mathrm{~km}$ over time intervals of 1 Jovian rotation. The zonal velocity $\bar{u}$ was constant to $1.5 \%$ during the 4 months between the Voyager 1 and 2 encounters. The latitudes of the zonal jet maxima (extrema of $\bar{u}$ ) are the same as inferred from earth-based observations extending over the past 80 years. The curvature of the velocity profile $d^{2} \bar{u} / d y^{2}$ varies with latitudinal coordinate $y$ in the range from $-3 \beta$ to $+2 \beta$, where $\beta$ is the planetary vorticity gradient. The barotropic stability criterion is violated at about 10 latitudes between $\pm 60^{\circ}$. The eddy momentum flux variation with latitude $\overline{u^{\prime} v^{\prime}}$ is positively correlated with $d \bar{u} / d y$ for both Voyager 1 and 2 data. The rate of conversion $\left\{K^{\top} K\right\}$ of eddy kinetic energy into zonal mean kinetic energy is in the range $1.5-3.0 \mathrm{Wm}^{-2}$, for a layer 2.5 bar deep. The time constant for resupply of zonal mean kinetic energy by eddies is in the range $2-4$ months, less than the interval between Voyager encounters. The rate of energy conversion is more than $10 \%$ of the total infrared heat flux for Jupiter, in contrast with earth where it is only $0.1 \%$ of the infrared heat flux. This hundred-fold difference suggests that the thermomechanical energy cycles are very different on the two planets.
\end{abstract}

\section{INTRODUCTION}

In earlier papers [Ingersoll et al., 1979; Beebe et al., 1980], we gave an analysis of wind vectors determined from Voyager 1 images at a resolution of $130 \mathrm{~km}$ (defined as twice the projected distance between adjacent picture elements, or pixels). Three important conclusions emerged: (1) The latitudes of the east-west jets have not changed during the past 80 years. (2) The peak zonal velocities are greater than suggested from earth-based observations. (3) The eddies are active and are transferring kinetic energy into the zonal jets at a large rate. The present paper extends these results to Voyager 2 images taken 4 months later.

We denote the eastward and northward velocity components by $u$ and $v$, respectively, with corresponding coordinates $x$ and $y$. Within a latitudinal bin $\left(1^{\circ}\right.$ wide in what follows) the longitudinal means are $\bar{u}$ and $\bar{v}$. The first result, inferred by comparing Voyager 1 images at $130 \mathrm{~km}$ resolution to 80 years of earth-based observations at $\geq 3000 \mathrm{~km}$ resolution, is that the latitudes and apparent speeds of the zonal jets do not change [Ingersoll et al., 1979; Beebe and Youngblood, 1979]. This constancy contrasts with the changing visual appearance of Jupiter from year to year, such that some currents become invisible from earth and some latitudes turn from light to dark from one year to the next. Thus it is interesting that no changes are observed in the zonal profiles $\bar{u}(y)$ between Voyager 1 and 2. The constancy of the zonal currents could be due to the great depth to which they extend, giving them a large inertia with respect to surface features [Ingersoll et al., 1979].

\footnotetext{
' Division of Geological and Planetary Sciences, California Institute of Technology, Pasadena, California 91125.

${ }^{2}$ Department of Astronomy, New Mexico State University, Las Cruces, New Mexico 88003.

${ }^{3}$ Earth and Space Sciences Division, Jet Propulsion Laboratory, California Institute of Technology, Pasadena, California 91103.

${ }^{4}$ Image Processing Laboratory, Jet Propulsion Laboratory, California Institute of Technology, Pasadena, California 91103.

${ }^{5}$ Laboratory for Planetary Atmospheres, University College, London, London WClE 6BT, England.

Copyright $\odot 1981$ by the American Geophysical Union.
}

The second result, that $\bar{u}$ is larger than expected, implies that the barotropic stability criterion $d^{2} \bar{u} / d y^{2} \leq \beta$ is violated on Jupiter [Ingersoll et al., 1979]. Here $\beta$ is the planetary vorticity gradient, $2 \Omega \cos \phi / r$, where $\Omega$ is the planetary rotation rate, $\phi$ is latitude, and $r$ is the planetary radius. Observations by Voyagers 1 and 2 show that $d^{2} \bar{u} / d y^{2}$ varies with latitude in the range from $-3 \beta$ to $+2 \beta$. Pre-Voyager theories [Rhines, 1975; Williams, 1978, 1979] of large-scale turbulence in rotating planetary atmospheres can explain the development of zonal jets from eddies, but the final state tends to satisfy $d^{2} \bar{u} /$ $d y^{2} \leq \beta$. In this paper we report on measurements of $d^{2} \bar{u} / d y^{2}$ from Voyager 1 and 2 and compare the measured values with $\beta$.

The third result involves the eddy wind components $u^{\prime}$ and $v^{\prime}$, defined as the departures of the individual wind vectors in a latitude bin from the longitudinal means $\bar{u}$ and $\bar{v}$. The root mean squared (rms) values of $u^{\prime}$ and $v^{\prime}$ for the bin are denoted by $\delta u$ and $\delta v$ and the cross correlation by $\overline{u^{\prime} v^{\prime}}$. The latter is the northward flux of eastward momentum per unit mass arising as a result of eddy motions. If $\overline{u^{\prime} v^{\prime}}$ were positive at latitudes where $d \bar{u} / d y$ is positive, and were negative where $d \bar{u} / d y$ is negative, then the eddy momentum flux would be toward latitudes where mean zonal momentum is greatest. The implied transfer rate of eddy kinetic energy to zonal mean kinetic energy per unit mass, which is given by the product $\bar{u}^{2} v^{\prime} \cdot d \bar{u} / d y$ averaged over latitude, would be positive. Multiplying this global integral by the mass per unit area involved in these motions, we obtain an estimate of the rate of eddy-to-mean-flow energy transfer per unit area. On earth this quantity is positive [Oort and Peixoto, 1974], but it is only $0.1 \%$ of the total thermal energy transfer (defined as the average emitted heat flux). On Jupiter this quantity is more than $10 \%$ of the total thermal energy transfer. Thus the thermomechanical energy cycle of Jupiter seems to be fundamentally different from that of earth.

\section{MEASUREMENT OF VeLocity}

We use the AMOS system at the Image Processing Laboratory of the Jet Propulsion Laboratory for determination of velocity. Errors arise both during feature identification and dur- 


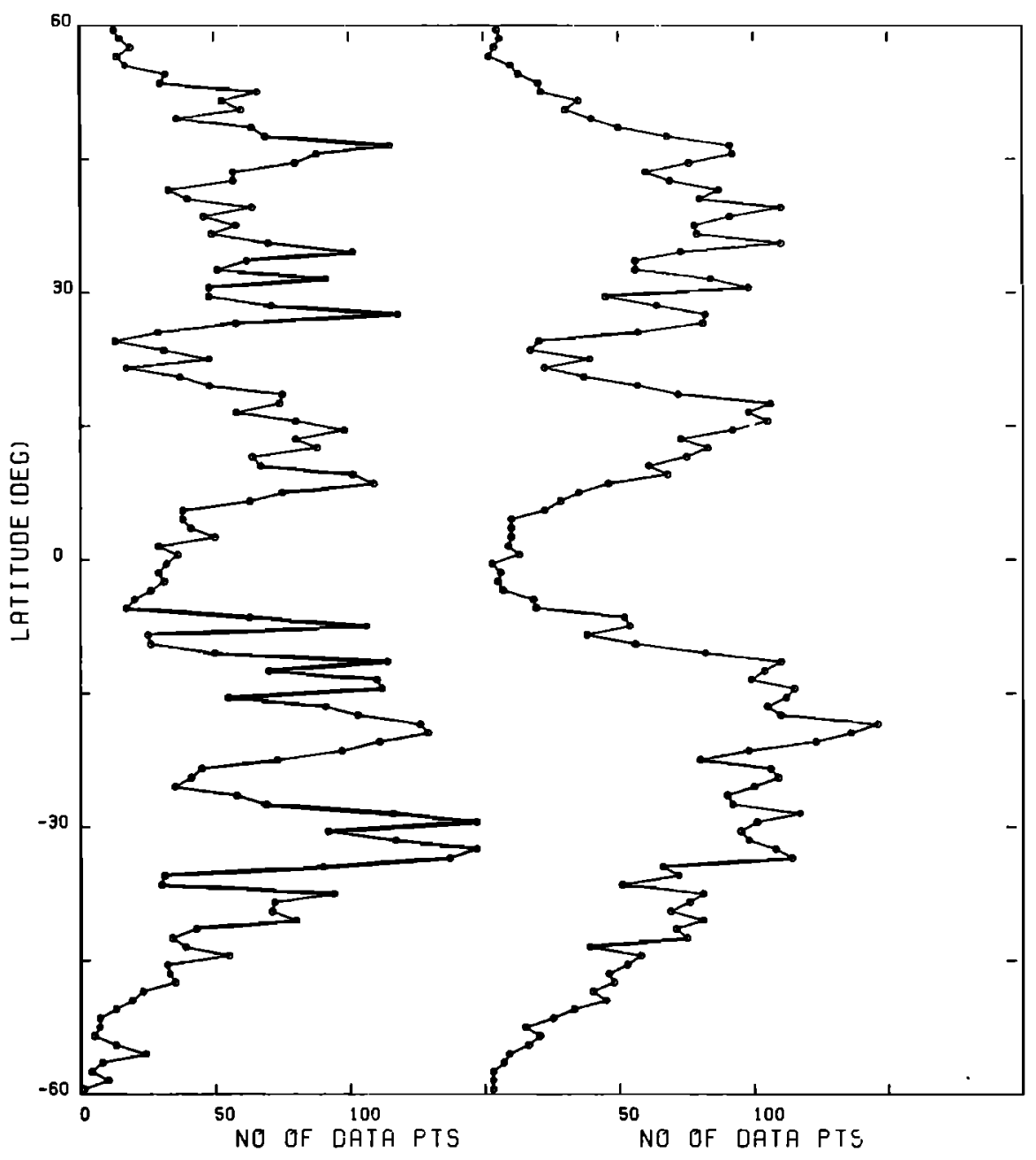

Fig. 1. The number of individual velocity vectors in each $1^{\circ}$ latitude bin for Voyager 1 and Voyager 2. Note the uneven sampling in latitude, owing to lack of long-lived features in some latitude bands. In all of these figures, the Voyager 1 curve is on the left and the Voyager 2 curve is on the right.

ing image navigation. The former procedure involves the operator's judgment in identifying the same feature in the two images and driving a cursor to the appropriate locations. The nature of the target-contrast, scale of features, variations in appearance during the time interval, etc.-affects the result. For good targets the resulting uncertainty in velocity, determined empirically, is approximately equal to the resolution (twice the distance between adjacent pixel locations) divided by the time between images. For the measurements reported here this uncertainty is about $\pm 3 \mathrm{~m} \mathrm{~s}^{-1}$ (resolution $\approx 130 \mathrm{~km}$, time separation $\approx 10$ hours $\approx$ one Jovian rotation).

The term image navigation refers to the precise determination of camera pointing. The time of shuttering, the spacecraft location with respect to Jupiter, and the camera orientation (rotation about the optic axis) are all known to sufficient accuracy. Camera pointing, however, is initially uncertain by as much as \pm 100 pixels $\left( \pm 9 \times 10^{-4}\right.$ radians) in the narrow angle frame. There are at least three ways to reduce this uncertainty: One either locates the limb in the narrow-angle frame, or one locates the limb in the simultaneously shuttered wide angle frame and transfers the information to the narrow angle frame, or one locates features whose positions are known. The first procedure is simplest and most reliable. The second, which was used for the measurements reported here, is less certain because the resolution of the wide angle camera is 7 times poorer. The third method gives only relative velocity and is used for high-resolution images where neither cameras (narrow or wide) saw the planetary limb. The second method gives a statistical error equivalent to two pixels divided by the time between images. For the measurements reported here, this is also about $\pm 3 \mathrm{~m} \mathrm{~s}^{-1}$. Systematic errors could arise owing to spacecraft attitude, spacecraft trajectory, and offsets of the narrow angle cameras with respect to the wide angle cameras. Such errors affect the absolute latitudes and longitudes more than they affect displacements, hence the systematic error in velocity is less than $\pm 2 \mathrm{~m} \mathrm{~s}^{-1}$.

We can estimate how these errors affect quantities such as $\bar{u}, d \bar{u} / d y, d^{2} \bar{u} / d y^{2}$, and $\overline{u^{\prime} v^{\prime}}$ as follows: Let $I$ be the number of measured features (wind vectors) in a latitude bin. Figure 1 shows the distributions of $I$ versus latitude for $1^{\circ}$ latitude bins for Voyagers 1 and 2 . Note that $I$ varies erratically from below 10 to over 100 , largely because some latitudes have more identifiable features than others. Feature identification contributes an error of about $\sigma_{I} / \sqrt{I}$ to the determination of $\tilde{u}$, where $\sigma_{I} \approx$ $3 \mathrm{~m} \mathrm{~s}^{-1}$ is the statistical error associated with feature identification. Navigation contributes an error of order $\sigma_{N} / \sqrt{N}$ where $\sigma_{N} \approx 3 \mathrm{~m} \mathrm{~s}^{-1}$ is the statistical error of navigation and $N$ is the number of frame pairs contributing to that bin. Since $N$ is typically in the range $10-20$, both sources of error may contribute, especially when $I$ is small. Figure 2 , showing $\bar{u}$ versus lati- 


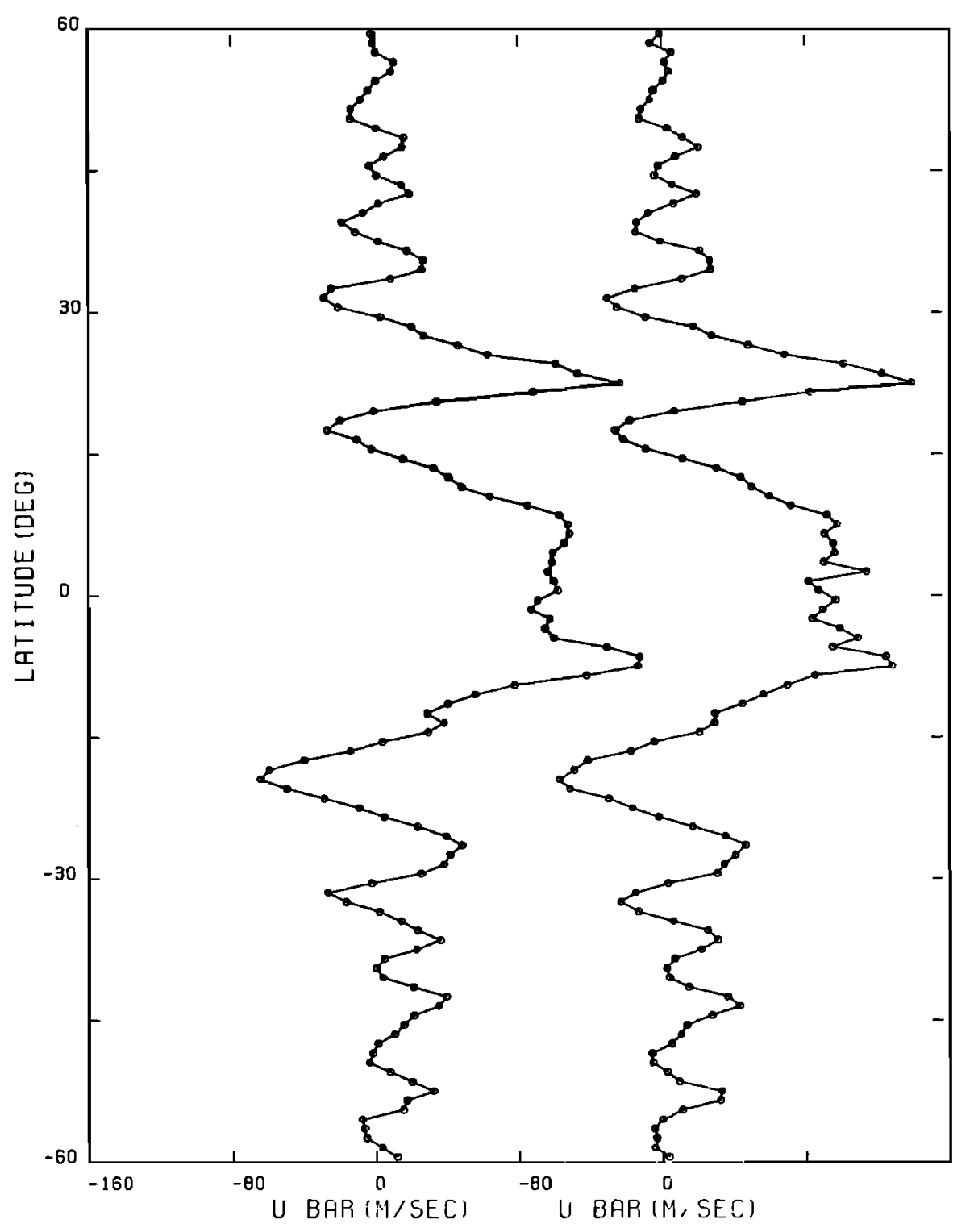

Fig. 2. Comparison of zonal velocity $\bar{u}$ in late February 1979 (Voyager 1, left) with that in early July 1979 (Voyager 2, right). The correlation coefficient is 0.986 for the two curves.

tude for Voyagers 1 and 2, reveals small-scale differences near the equator and near $\pm 60^{\circ}$ latitude (planetographic latitudes are used throughout). These are the regions where $I \leq 10$, according to Figure 1, and where $\sigma_{I}$ is larger than $3 \mathrm{~m} \mathrm{~s}^{-1}$ owing to the difficulty of identifying features. We attribute such differences between Voyagers 1 and 2 to statistical error.

We compute $d \bar{u} / d y$ and $d^{2} \bar{u} / d y^{2}$ at latitudinal position $y_{n}$ as follows:

$$
\begin{gathered}
(d \bar{u} / d y)_{n}=\left(\bar{u}_{n+1}-\bar{u}_{n-1}\right)(\Delta y)^{-1} \\
\left(d^{2} \bar{u} / d y^{2}\right)_{n}=\left(\bar{u}_{n+2}+\bar{u}_{n-2}-2 \bar{u}_{n}\right)(\Delta y)^{-2}
\end{gathered}
$$

where

$$
\Delta y=y_{n+1}-y_{n-1}
$$

Both formulas tend to smooth out irregular variations (e.g., near the equator in Figure 2) that we attribute to feature identification error. The error in $d \bar{u} / d y$ is then about $\sqrt{2} \sigma(\bar{u}) / \Delta y$ and that in $d^{2} \bar{u} / d y^{2}$ about $\sqrt{6} \sigma(\bar{u}) /(\Delta y)^{2}$, where $\sigma(\bar{u})$ is the statistical error in $\bar{u}$ (assumed uncorrelated between latitudes). Figures 3 and 4 show $d \bar{u} / d y$ and $d^{2} \bar{u} / d y^{2}$, respectively. Again the error, identified with small-scale irregular variations having no correlation between Voyagers 1 and 2, is concentrated at latitudes where the number of identifiable features is small (Figure 1). Figure 5 shows $\bar{v}$ versus latitude. The means and variance of $\bar{v}$ provide a measure of systematic error. Averaging with respect to latitude we obtain $\langle\bar{\nu}\rangle=0.6 \pm 2.3 \mathrm{~m} \mathrm{~s}^{-1}$ for Voyager 1 and $(\bar{v})=0.9 \pm 1.3 \mathrm{~m} \mathrm{~s}^{-1}$ for Voyager 2 .

The eddy cross correlation $\overline{u^{\prime} v^{\prime}}$, the rms eddy velocities $\delta u$ and $\delta v$, and the correlation coefficient $r\left(u^{\prime}, v^{\prime}\right)$ are computed for each bin from the formulas

$$
\begin{gathered}
\overline{u^{\prime} v^{\prime}}=\frac{1}{I} \sum_{i=1}^{I}\left(u_{i}-\bar{u}\right)\left(v_{i}-\bar{v}\right) \\
(\delta u)^{2}=\frac{1}{I} \sum_{i=1}^{L}\left(u_{i}-\bar{u}\right)^{2} \\
(\delta v)^{2}=\frac{1}{I} \sum_{i=1}^{l}\left(v_{i}-\bar{v}\right)^{2} \\
r\left(u^{\prime}, v^{\prime}\right)=\overline{u^{\prime} v^{\prime}}(\delta u \delta v)^{-1}
\end{gathered}
$$




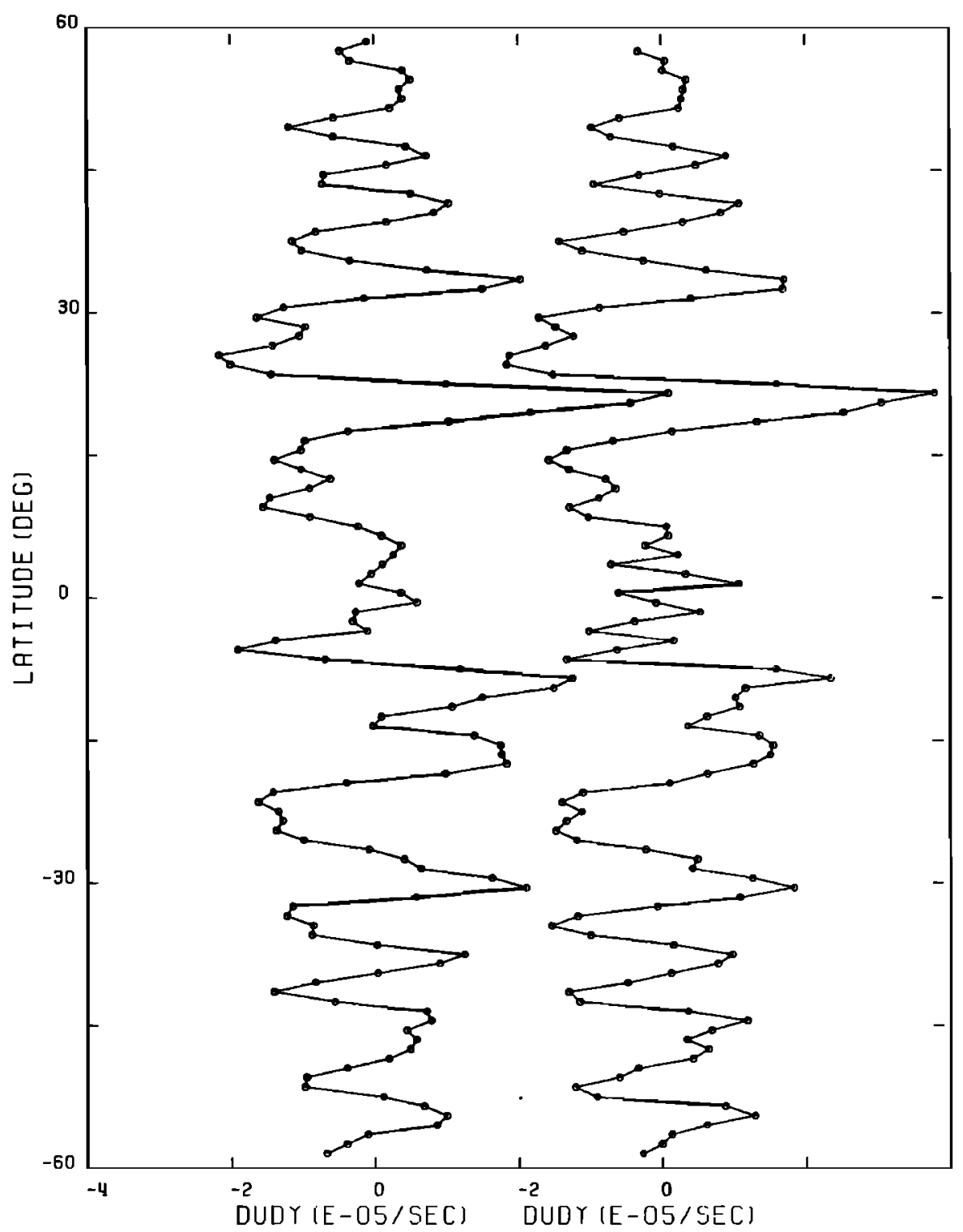

Fig. 3. Same as Figure 2 except $d \bar{u} / d y$ is plotted.

Each $u_{i}-\bar{u}$ is an individual measurement of the velocity fluctuation $u^{\prime}$ and consists of two parts: the true eddy component with rms amplitude $\delta u_{e}$ and the statistical measurement error with rms amplitude $\sigma_{u}$ (the corresponding quantities for $v_{i}-\bar{v}$ are $\delta v_{e}$ and $\sigma_{v}$ ). Thus $\delta u^{2}=\delta u_{e}^{2}+\sigma_{u}^{2}$ and $\delta v^{2}=\delta v_{e}^{2}+\sigma_{p}^{2}$. The variance of $\frac{u^{\prime} v^{\prime}}{\text { is given by }}$

$$
\operatorname{var}\left(\overline{u^{\prime} v^{\prime}}\right)=\left(\delta u_{e}^{2} \sigma_{v}{ }^{2}+\sigma_{u}{ }^{2} \delta v_{e}{ }^{2}+\sigma_{u}{ }^{2} \sigma_{v}{ }^{2}\right) / I
$$

provided the errors in $u$ and $v$ are uncorrelated. Since $\sigma_{u}$ and $\sigma_{v}$ are both in the range 4 to $8 \mathrm{~m} \mathrm{~s}^{-1}, \delta u$ is $10-20 \mathrm{~m} \mathrm{~s}^{-1}$, and $\delta v$ is 5-10 $\mathrm{m} \mathrm{s}^{-1}$ (Figures 6 and 7), the main contribution to the variance is from the first term. Thus the rms error in $\overline{u^{\prime} v^{\prime}}$ is of order $\delta u \sigma_{v} / \sqrt{I}$. In the best cases, when $\delta u \approx 10 \mathrm{~m} \mathrm{~s}^{-1}, \sigma_{v} \approx 4 \mathrm{~m}$ $\mathrm{s}^{-1}$, and $I \approx 120$, the rms error of $\overline{u^{\prime} v^{\prime}}$ is of order $4 \mathrm{~m}^{2} \mathrm{~s}^{-2}$. In the worst cases the rms error may be 15 times larger. This is consistent with the curves of Figures 8 and 9 showing $\overline{u^{\prime} v^{\prime}}$ and $r\left(u^{\prime}, v^{\prime}\right)$ as functions of latitude.

\section{Results of DATA ANALYSIS}

The Voyager 1 data are the same as discussed by Beebe et al. [1980], and cover the period around February 26-27, 1979. The Voyager 2 data cover July 1-2, 1979. Most frame pairs are 10 hours apart, although some are 20 hours apart. The images were part of a sequence of $3 \times 3$ mosaics covering the disk at about seven equally spaced longitudes. There are 53 frame pairs for Voyager 1 and 25 for Voyager 2. The total number of individual vectors is 6933 for Voyager 1 and 7177 for Voyager 2. About $0.7 \%$ of the data (60 and 33 vectors, respectively) were discarded for having $u^{\prime}$ or $v^{\prime}$ greater than 150 $\mathrm{m} \mathrm{s}^{-1}$ (a limit of $50 \mathrm{~m} \mathrm{~s}^{-1}$ was used from $19^{\circ}$ to $25^{\circ}$ latitude).

The results discussed below are obtained from analysis of the latitudinal profiles of Figures 1-9. Individual velocity vectors enter only as they contribute to the longitudinal means $\vec{u}$, $\bar{v}, \overline{u^{\prime} v^{\prime}}$, etc. We define the correlation coefficient $r(\bar{p}, \bar{q})$, where $\bar{p}(y)$ and $\bar{q}(y)$ are longitudinal mean quantities, as that computed from $N$ pairs of numbers $(p, q)$, where $N$ is the number of latitude bins from $-60^{\circ}$ to $+60^{\circ}$. We exclude latitudes where the number of data points $I$ is less than 10 . For intercomparisons of Voyager 1 and Voyager 2 data this gives $N=$ 103. For Voyager 1 intracomparisons $N=114$, and for Voyager 2 intracomparisons $N=106$.

The correlation coefficient $r\left(\bar{u}_{1}, \bar{u}_{2}\right)$ for comparing the Voyager 1 and Voyager 2 zonal velocity profiles (Figure 2) is 0.986 . The correlation coefficients for $d \bar{u} / d y$ and $d^{2} \bar{u} / d y^{2}$ are 


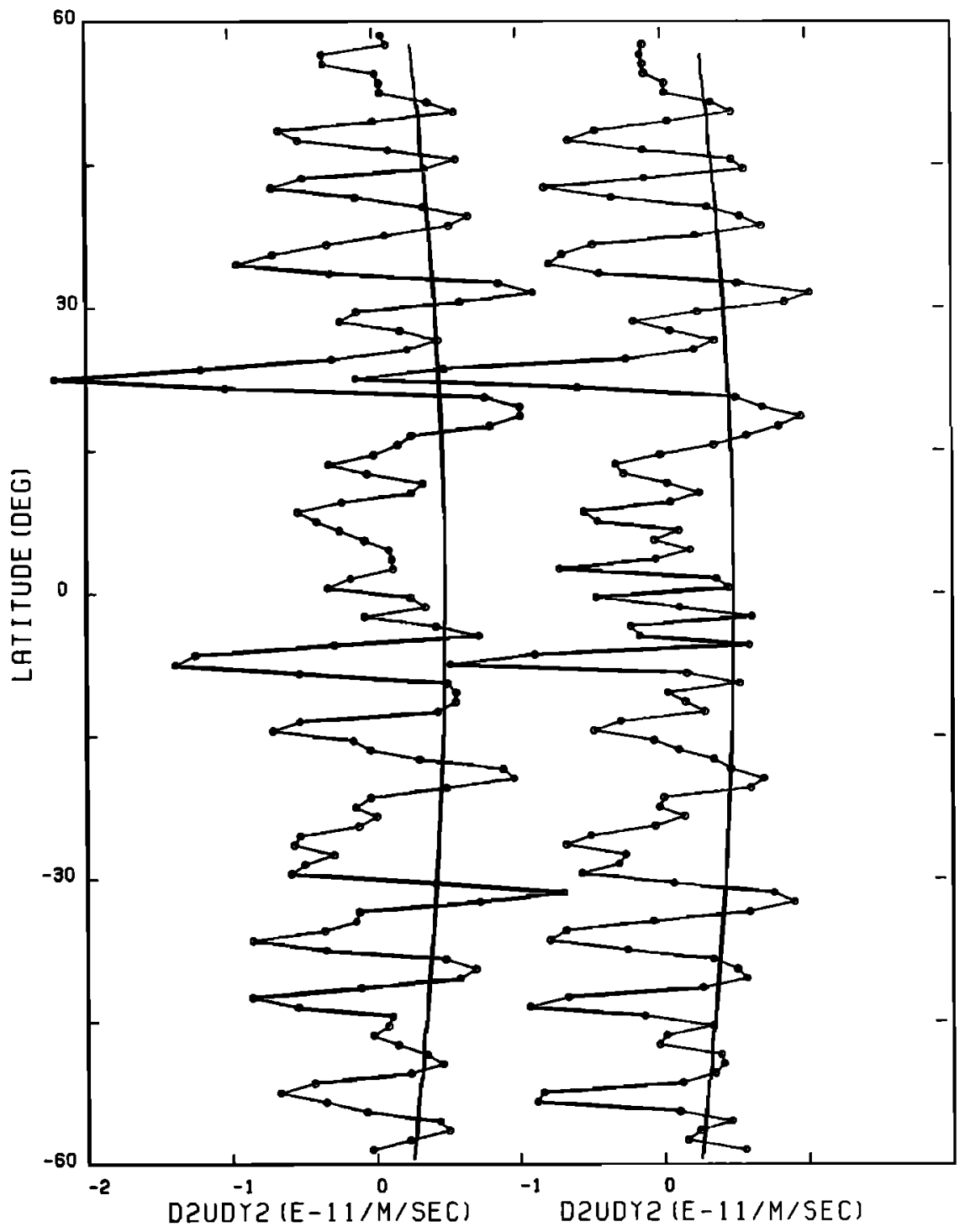

Fig. 4. Same as Figure 2 except $d^{2} \tilde{u} / d y^{2}$ is plotted. The smooth curves give $\beta$, the planetary vorticity gradient, in the same units.

0.940 and 0.880 , respectively. Although only 103 latitudes contribute to the correlation coefficients, other latitudes with fewer than 10 points contribute to the derivatives (equations (1) and (2)). From these results and from close inspection of Figures $2-4$, we conclude that the zonal velocity profile $\bar{u}(y)$ did not change significantly during the 4 months between encounters.

Table 1 shows a comparison of these Voyager velocity profiles with 80 years of earth-based observation, as summarized by Smith and Hunt [1976]. We give only the latitudes at which the zonal velocity profile $\bar{u}(y)$ attains extreme values. These zonal jet maxima are easy to identify in Figure 2. We have not included the small inflections in the curves near $\pm 13^{\circ}$ latitude, which we believe to be real, or the irregular variations near the equator, which we believe are due to the small numbers of trackable features at these latitudes. The names of the currents are from Smith and Hunt's review. Other traditional currents that lie between zonal jet maxima are not included in our table. Comparing columns $1-4$, it is clear that the latitudes of the zonal jet maxima have changed very little during $\mathbf{8 0}$ years. All the traditional currents were seen by both Voyagers.
To quote Smith and Hunt [1976, p. 565], 'although some temporal variation in velocity is observed in all zonal currents, each latitude of Jupiter is characterized by a particular zonal motion that remains relatively constant over many decades.'

Another feature of the zonal velocity profile is its northsouth symmetry. In each hemisphere there are seven zonal jet maxima between $0^{\circ}$ and $45^{\circ}$ latitude. The correspondence in latitude between the two hemispheres is not perfect; the phases of the patterns in north and south differ by as much as one-quarter cycle, and the magnitudes of the velocities differ by factors of 2 . Still, the degree of symmetry is good up to $43^{\circ}$. Above this latitude there is less correspondence between the two hemispheres.

The velocity magnitudes given in Table 1 are from Voyager. These are systematically higher, especially for the westward jets and especially at high north and south latitudes, than those inferred from earth-based observations. One new inference from Voyager is that the barotropic stability criterion $d^{2} \bar{u} / d y^{2} \leq \beta$ is violated at the latitudes of the westward jets [Ingersoll et al., 1979]. This fact is apparent in Figure 4, in which $d^{2} \bar{u} / d y^{2}$ and $\beta$ are plotted on the same scale for Voy- 


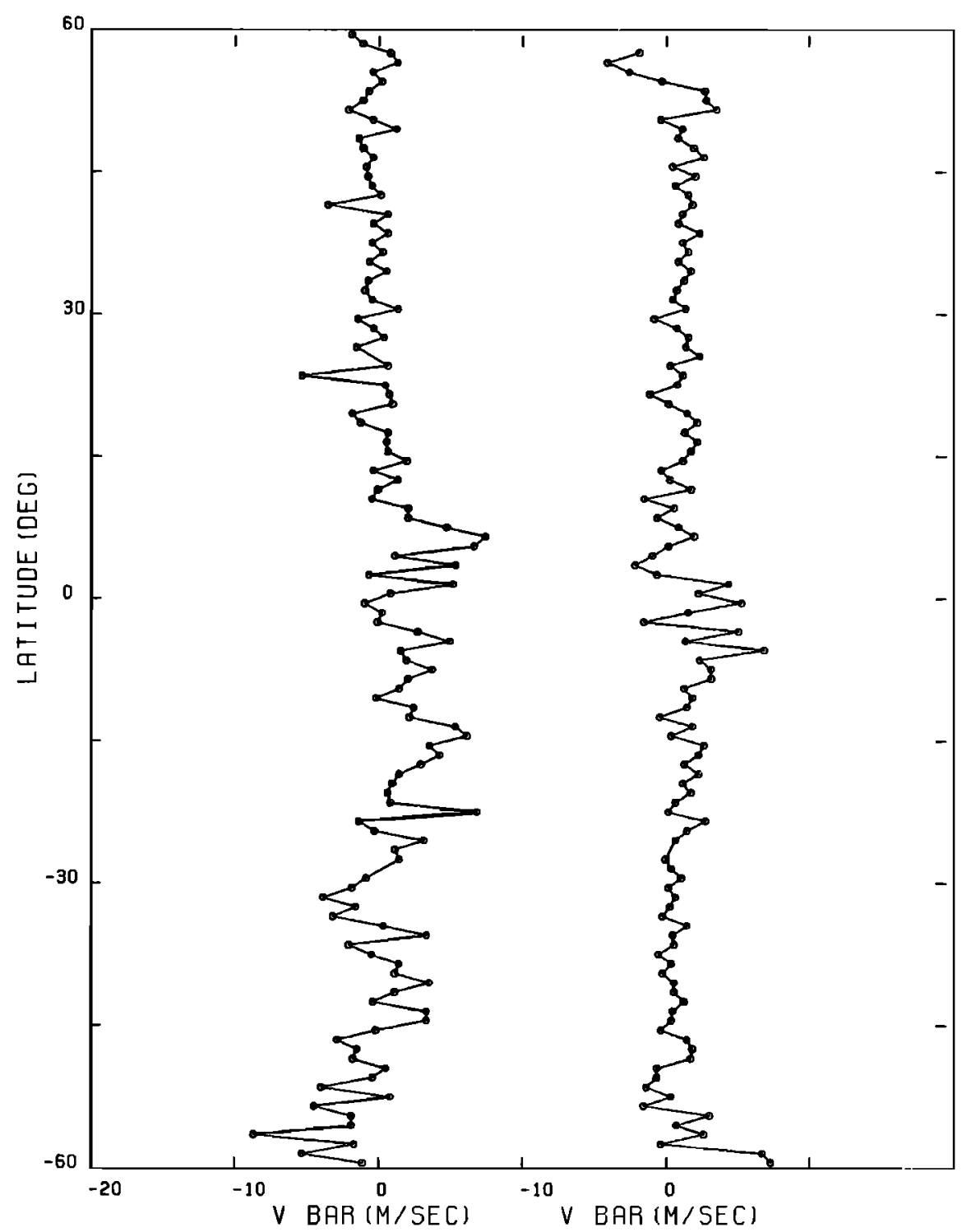

Fig. 5. Same as Figure 2 except the mean northward velocity $\bar{v}$ is plotted.

ager 1 and 2. One observes that $d^{2} \bar{u} / d y^{2}$ varies between $-3 \beta$ and $+2 \beta$ as a function of latitude. Estimates based on earthbased data [Ingersoll and Cuzzi, 1969] seem to have underestimated $d^{2} \bar{u} / d y^{2}$ by a factor of 2 .

Figure 8 shows $\overline{u^{\prime} v^{\prime}}$ for Voyagers 1 and 2 compared with $d \bar{u}$ / $d y$ for Voyager 1. Figure 9 shows $r\left(u^{\prime}, v^{\prime}\right)$ compared with $d \bar{u}$ / $d y$. Recall that the correlation coefficient of $d \bar{u} / d y$ for Voyager 1 with $d \bar{u} / d y$ for Voyager 2 is 0.94 . The variations of $d \bar{u} / d y$ versus latitude are therefore not random, but reflect instead the zonal current structure of Jupiter. In contrast, the correlation coefficient of $\overline{u^{\prime} v^{\prime}}$ for Voyager 1 with $\overline{u^{\prime} v^{\prime}}$ for Voyager 2 is 0.36, for 103 latitudes that have 10 or more points. And the correlation coefficients of $\overline{u^{\prime} v^{\prime}}$ with $d \bar{u} / d y$ for Voyager 1 , and $\overline{u^{\prime} v^{\prime}}$ with $d \bar{u} / d y$ for Voyager 2 , are 0.46 and 0.30 , respectively. Thus $\overline{u^{\prime} v^{\prime}}$ behaves like a random variable, although it is significantly correlated with itself (for a 4-month lag) and with dü/ $d y$. Similar remarks apply to $r\left(u^{\prime}, v^{\prime}\right)$. The correlation coefficient for 103 latitude bins of $r\left(u^{\prime}, v^{\prime}\right)$ for Voyager 1 with $r\left(u^{\prime}\right.$, $\left.v^{\prime}\right)$ for Voyager 2 is 0.33 , and the correlation coefficients of $r\left(u^{\prime}, v^{\prime}\right)$ with $d \bar{u} / d y$ for Voyagers 1 and 2 are 0.53 and 0.46 , respectively.
Two normally distributed random variables, $x$ and $y$, are significantly correlated at the $1 \%$ level when $r(x, y) \geq 0.325$ and $r \geq 0.254$, for 60 and 100 degrees of freedom (DF), respectively. Since there are at least 100 latitudes where $I \geq 10$, a value of $r>0.4$ is highly significant. One reaches the same conclusion if instead of computing a correlation coefficient one solves for $A$ and $B$ and their rms errors $\sigma(A)$ and $\sigma(B)$ in the equations

$$
\begin{gathered}
\overline{u^{\prime} v^{\prime}}=A d \bar{u} / d y+C \\
r\left(u^{\prime}, v^{\prime}\right)=B d \bar{u} / d y+D
\end{gathered}
$$

using the method of least squares. To a good approximation one finds

$$
\begin{gathered}
A / \sigma(A) \approx N^{1 / 2} r\left(\overline{u^{\prime} v^{\prime}}, d \bar{u} / d y\right) \\
B / \sigma(B) \approx N^{1 / 2} r\left[\left(u^{\prime}, v^{\prime}\right), d \bar{u} / d y\right]
\end{gathered}
$$

where $N$ is the number of latitude bins, as before. The ratio $A / \sigma(A)$ is in the range 3-5 for our data, and the ratio $B / \sigma(B)$ is in the range 5-7. Again, this is significant at the $1 \%$ level. Use of regression equations such as (8) and (9) avoids the assump- 


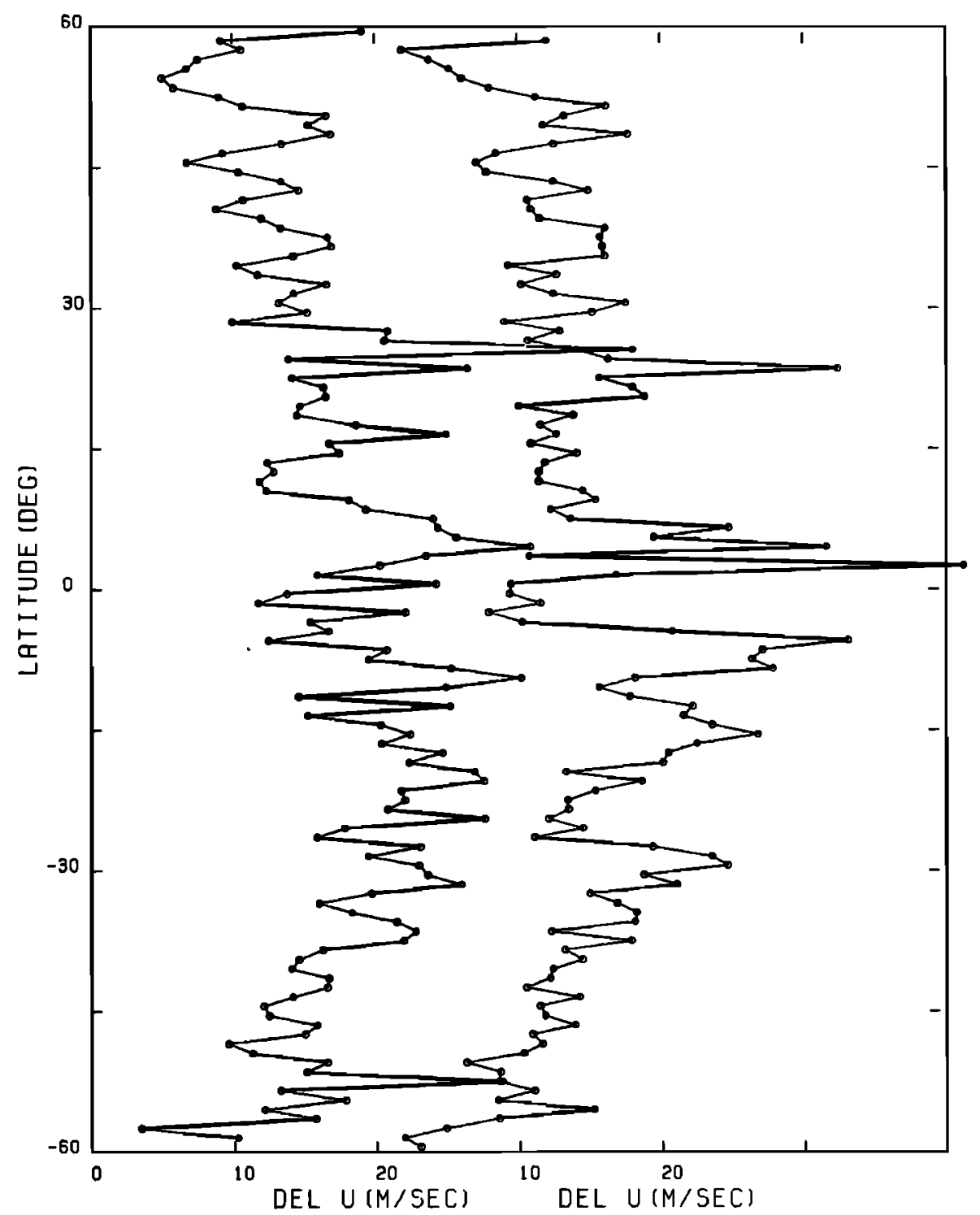

Fig. 6. Same as Figure 2 except the rms eastward eddy component $\delta u$ is plotted.

tion that $d \bar{u} / d y$ is normally distributed. By using a $t$ test, values of $A / \sigma(A) \geq 4$ are significant at the $1 \%$ level as long as DF $>5$, and values of $A / \sigma(A) \geq 2.6$ are significant at the $1 \%$ level for $\mathrm{DF}>100$.

The assumption, DF = number of latitude bins, is valid so long as feature identification errors dominate errors of navigation. Then each measurement is independent, and the errors between latitude bins are uncorrelated. When errors of navigation dominate, DF is given by the number of frame pairs contributing. In either case, the relation between $\overline{u^{\prime} v^{\prime}}$ and $d \bar{u}$ / $d y$ is statistically significant. If the eddies were spatially resolved by our measurements, DF might be smaller than either of the above estimates. However, the typical 'blob' apparent in the images has a diameter of $1000 \mathrm{~km}$ or less. Movie sequences suggest that these blobs are genuine flow featureseddies. Our velocity vectors are spaced irregularly, but typically they are $2000 \mathrm{~km}$ apart (7000 vectors over the surface of the planet). Thus our measurements refer to separate eddies and are therefore statistically independent.

Two aspects of Figures 1-9 remain unexplained. First, the correlation between $r\left(u^{\prime}, v^{\prime}\right)$ and $d \bar{u} / d y$ is better than that be- tween $\overline{u^{\prime} v^{\prime}}$ and $d \bar{u} / d y$. Apparently, the correlation between $u^{\prime}$ and $v^{\prime}$ is present even at latitudes where the rms magnitudes $\delta u$ and $\delta v$ are relatively small. Dividing $\overline{u^{\prime} v^{\prime}}$ by $\delta u \delta v$ to get $r\left(u^{\prime}\right.$, $\left.v^{\prime}\right)$ tends to bring out this correlation. Figure 9 shows that the correlation between $u^{\prime}$ and $v^{\prime}$ tends to have the same sign as $d \bar{u} / d y$, indicating momentum transport by eddies into the zonal jets.

Second, the nonzonal and eddy velocity components $\delta v, \bar{v}$, and $\left(\overline{u^{\prime} v^{\prime}}\right)^{1 / 2}$, are smaller for Voyager 2 than for Voyager 1. Movie sequences and selected high-resolution frames suggest that Jupiter was just as active in July 1979 as it was in March 1979. Differences may have arisen during data processing. First, the need for simultaneous wide-angle coverage dictated that orange narrow-angle frames be used for Voyager 1 feature tracking and violet narrow-angle frames for Voyager 2. Also, fewer frame pairs (25 versus 53) were used for Voyager 2 compared with Voyager 1. Finally, different individuals did the feature tracking for the two spacecraft. Those who contributed most to the Voyager 2 data set may have chosen the most reliable features, thereby biasing the data away from the turbulent, active regions of Jupiter. Or, the Voyager 1 data set 


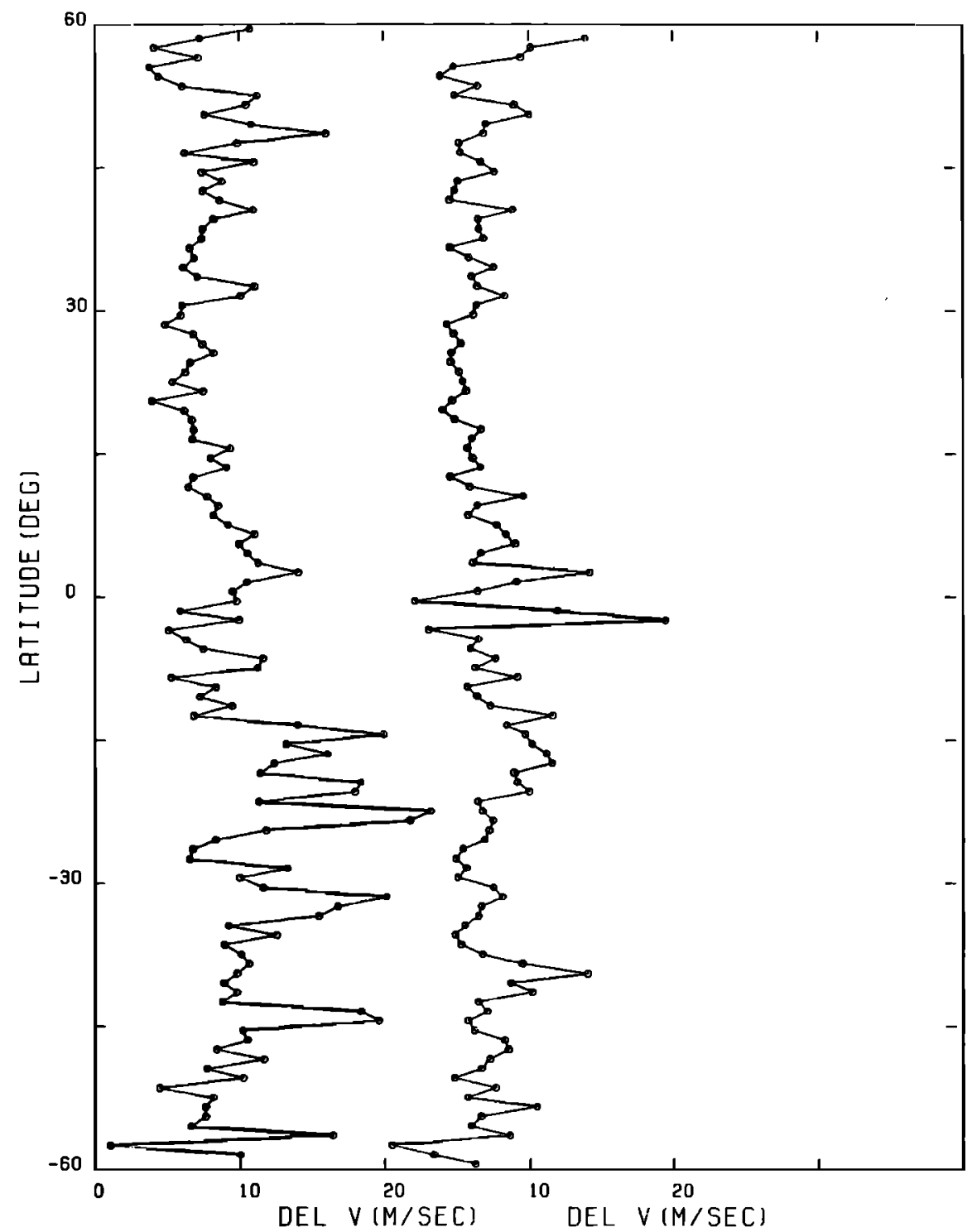

Fig. 7. Same as Figure 2 except the rms northward eddy component $\delta v$ is plotted.

may be biased in the opposite way. There is some indication in Figure 1 of a difference in latitudinal sampling between the two spacecraft. Such a difference might be due either to color differences or to different individual sampling strategies. Such bias will be assessed and corrected if possible in future analyses of Voyager data.

\section{Discussion AND CONCLUSION}

We have seen that the mean zonal velocity profile $\bar{u}(y)$ did not change by a measureable amount between Voyagers 1 and 2 (Figure 2). This is consistent with the observation that every major current that has ever been seen from earth was seen by Voyager at its expected latitude (Table 1). Apparently, the zonal currents are more steady than the visible clouds, suggesting that they extend into Jupiter's adiabatic fluid interior well below the clouds. Their steadiness might be due to their huge inertia if they extend deep enough.

We have also seen that $d^{2} \bar{u} / d y^{2}$ varies with latitude in the range $-3 \beta$ to $+2 \beta$ (Figure 4). Pre-Voyager analysis of earthbased data [Ingersoll and Cuzzi, 1969] gave $d^{2} \bar{u} / d y^{2} \leq \beta$, apparently because of the tendency of large spots to roll between zonal jets and not partake in the extreme flow [Ingersoll et al.,
1979]. Numerical experiments [Rhines, 1975; Williams, 1978, 1979] with eddy-mean-flow interaction show that both stratified and unstratified rotating fluids tend to relax to a state in which the flow is mostly zonal and $d^{2} \tilde{u} / d y^{2} \leq \beta$. The numerical experiments have been run with a variety of forcings and initial conditions, including mechanical forcing and thermal (baroclinic) forcing. The computed flows seem to satisfy marginally the barotropic stability criterion $d^{2} \bar{u} / d y^{2} \leq \beta$. Whether a factor of 2 discrepancy is theoretically significant remains to be seen. The numerical experiments should be rerun with the Voyager observation $-3 \beta \leq d^{2} \bar{u} / d y^{2} \leq 2 \beta$ in mind.

Finally, we have seen that $\overline{u^{\prime} v^{\prime}}$ and $r\left(u^{\prime}, v^{\prime}\right)$ as functions of latitude are statistically related to $d \bar{u} / d y$ (Figures 8 and 9). The correlation coefficients tend to be $0.4-0.5$, indicating that most $\left(\approx 1-r^{2}\right)$ of the variance of $\overline{u^{\prime} v^{\prime}}$ is not associated with $d \bar{u} / d y$. Measurement error and Jovian atmospheric flows might both contribute to this uncorrelated variance.

Following Beebe et al. [1980] we have computed values of the coefficient $A$ in (8), as well as values of $\left\{K^{\prime} \bar{K}\right\}$, where

$$
\left\{K^{\prime} \bar{K}\right\}=\frac{1}{N} \sum_{n=1}^{N}\left|\frac{d \bar{u}}{d y}\right|_{n}\left(\overline{u^{\prime} v^{\prime}}\right)_{n}
$$




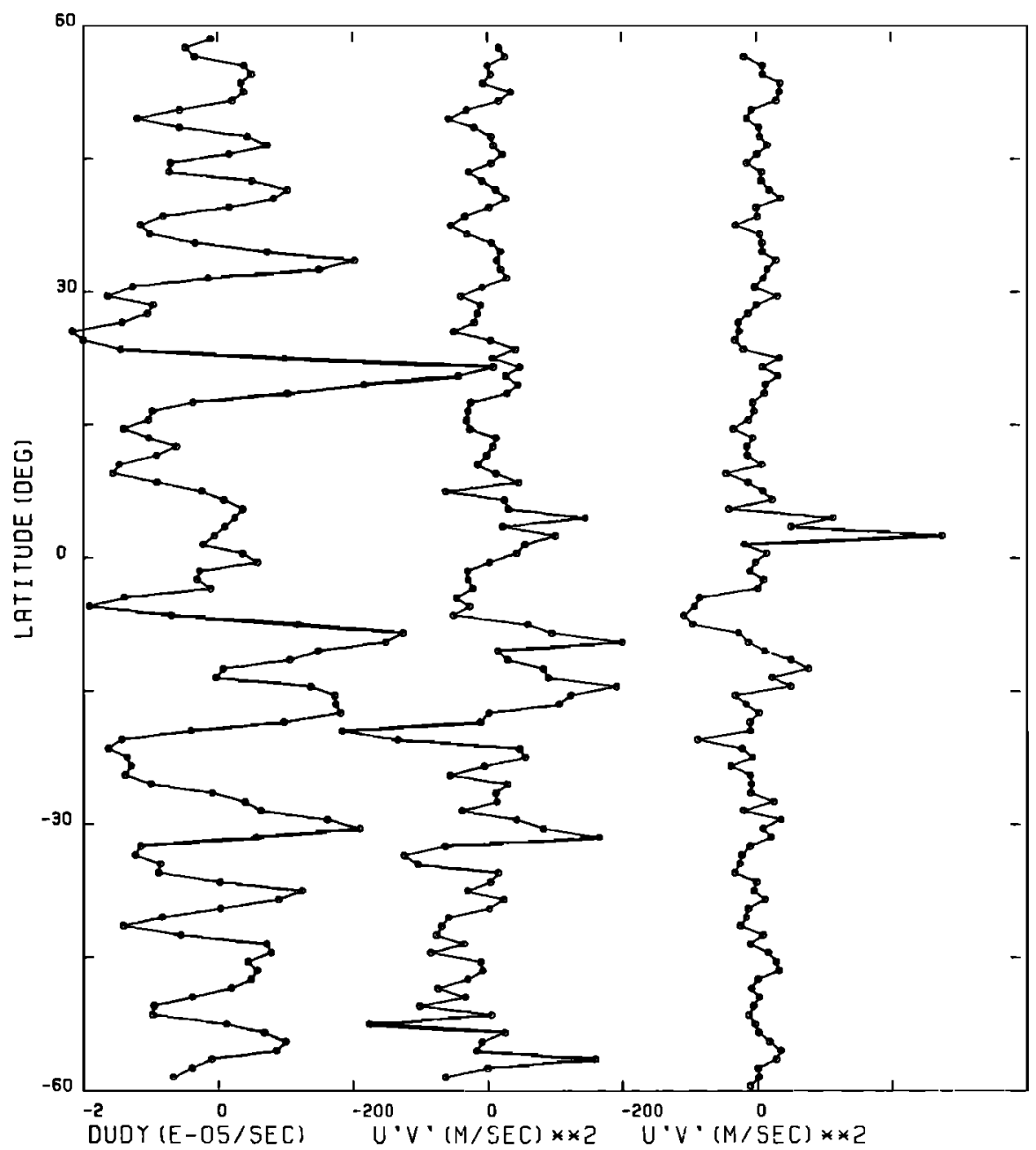

Fig. 8. The northward eddy transport of eastward momentum $\overline{u^{\prime} v^{\prime}}$ (Voyager 1, center, and Voyager 2, right) compared to $d \bar{u} / d y$ for Voyager 1 (left). A positive correlation indicates that eddies are transferring kinetic energy into the mean zonal flow $u(y)$. The implied rate of energy transfer relative to thermal energy transfer is 0.1 on Jupiter, 0.001 on earth.

TABLE 1. Latitudes of Zonal Jet Maxima

\begin{tabular}{|c|c|c|c|c|c|}
\hline \multirow[b]{2}{*}{ Name of Current } & \multicolumn{4}{|c|}{ Latitude (deg)* } & \multirow[b]{2}{*}{$u(\mathrm{~m} / \mathrm{s}) \dagger$} \\
\hline & 1 & 2 & 3 & 4 & \\
\hline North polar region & & & & 56.6 & 10 \\
\hline North polar region & & & & 51.0 & -13 \\
\hline North polar region & & & & 47.5 & 20 \\
\hline North polar region & & & & 45.0 & -4 \\
\hline N.N.N. temperate current & 43 & $44-46$ & $42.8-45.9$ & 43.0 & 19 \\
\hline N.N. temperate current $A$ & $36-40$ & $35-41$ & $37.3-40.6$ & 39.0 & -19 \\
\hline N.N. temperate current $B$ & 35 & $\cdots \neq$ & $35.1-35.8$ & 35.0 & 27 \\
\hline N. temperate current $A$ & $29-33$ & $28-32$ & $30.2-31.4$ & 31.5 & -31 \\
\hline N. temperate current $C$ & 23 & $\cdots \neq$ & $23.8-24.2$ & 23.0 & 138 \\
\hline N. tropical current $A$ & 14-22 & $14-21$ & $15.6-19.6$ & 17.5 & -26 \\
\hline N. equatorial current & $3-10$ & $4-8$ & $6.6-8.6$ & 7.0 & 102 \\
\hline Central equatorial current & & & & 0.0 & 95 \\
\hline S. equatorial current & $3-10$ & $6-8$ & $5.8-7.6$ & 7.0 & 137 \\
\hline S. edge $\mathrm{SEB}_{s}$ & 19 & $18-22$ & $20.3-21.7$ & 19.5 & -61 \\
\hline N. edge STB & 27 & 26 & $25.2-26.2$ & 26.5 & 47 \\
\hline S. temperate current & 29 & $32-35$ & $33.6-33.7$ & $\begin{array}{l}32.0 \\
36.5\end{array}$ & $\begin{array}{r}-25 \\
34\end{array}$ \\
\hline \multirow[t]{3}{*}{ S.S. temperate current } & $38-445$ & $39-45$ & $38.8-41.3$ & 39.5 & 1 \\
\hline & & & & 43.0 & 41 \\
\hline & & & & 49.0 & -3 \\
\hline South polar region & & & & 49.0 & -3 \\
\hline South polar region & & & & 52.5 & 33 \\
\hline South polar region & & & & 56.5 & -6 \\
\hline
\end{tabular}

"Columns 1, 2, 3 are from Smith and Hunt [1976] and cover the years 1898-1948, 1946-1964, 1962-1970, respectively. Column 4 is from Voyager (this work), and covers the first half of 1979.

†Magnitude of the zonal velocity $u$ is from Voyager (this work).

$\ddagger$ Indicates that the current was not observed during the time interval. 


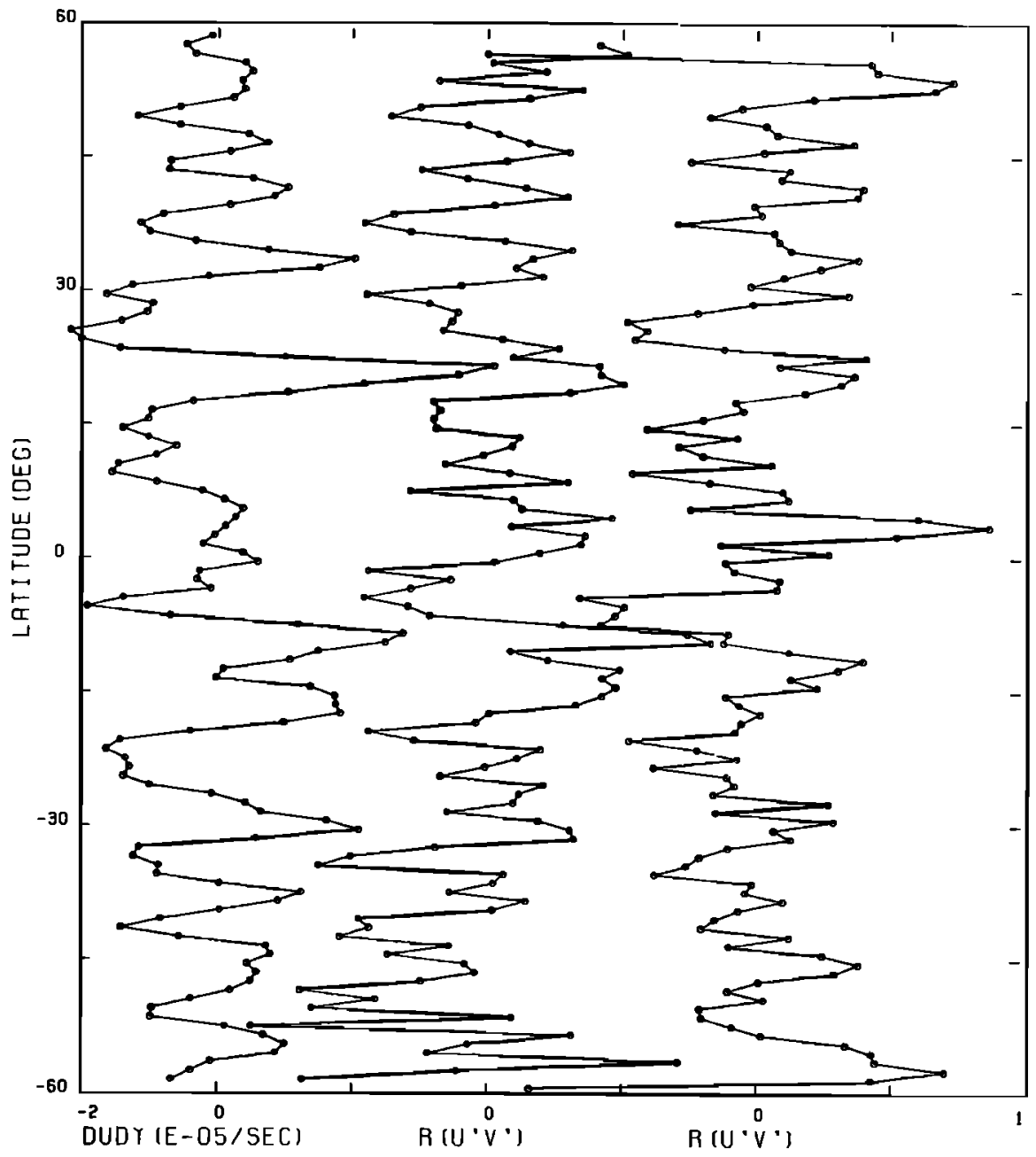

Fig. 9. Same as Figure 8 except the eddy correlation coefficient $r\left(u^{\prime}, v^{\prime}\right)$ is plotted.

$N$ being the number of latitude bins. Values of $A$ are the same as negative viscosity coefficients. For these Jupiter data they range from $1.3 \times 10^{6}$ to $2.6 \times 10^{6} \mathrm{~m}^{2} \mathrm{~s}^{-1}$. With the velocity data reported here we cannot resolve the scales of the flow structures contributing to $A$. For eddy velocities of order $10 \mathrm{~m}$ $\mathrm{s}^{-1}$, which is a typical rms value of $\delta u$ or $\delta v$ (Figures 6 and 7), the length scales would have to be at least $200 \mathrm{~km}$ to give values of $A$ around $2 \times 10^{6} \mathrm{~m}^{2} \mathrm{~s}^{-1}$. Careful study of some large eddies [Mitchell et al., this issue] should help isolate their contributions to $\overline{u^{\prime} v^{\prime}}$.

As was discussed by Beebe et al. [1980], the values of $\left\{K^{\prime} \bar{K}\right]$ implied by these data are large. Here $\left\{K^{\prime} \bar{K}\right\}$ is the average rate per unit mass at which eddy kinetic energy is converted to zonal mean kinetic energy. Typical values are $(1.5-3.0) \times$ $10^{-4} \mathrm{~m}^{2} \mathrm{~s}^{-3}$. As with equations (10) and (11), the value of $\left\{K^{\prime} \bar{K}\right\}$ divided by its standard deviation is $N^{1 / 2} r\left(\overline{u^{\prime} v^{\prime}}, d \bar{u} / d y\right)$ to a good approximation. For these data, this quantity is between 3 and 5 , implying that $\left\{K^{\prime} \bar{K}\right\}$ is also significantly different from zero. Multiplying $\left\{K^{\prime} \bar{K}\right\}=(1.5-3.0) \times 10^{-4} \mathrm{~m}^{2} \mathrm{~s}^{-3}$ by $10^{4} \mathrm{~kg} \mathrm{~m}^{-2}$, the mass per unit area in layer 2.5 bar thick on Jupiter, we derive $(1.5-3.0) \mathrm{Wm}^{-2}$ as the power per unit area. If we assume the eddy cross correlation extends deeper than 2.5 bars, the power per unit area will be proportionately higher. We choose $10^{4} \mathrm{~kg} \mathrm{~m}^{-2}$ as a convenient round number and a conservative (low) estimate of the mass between the optical cloud tops $(P \approx 1$ bar) and the computed base of the wa- ter cloud according to solar composition models [Weidenschilling and Lewis, 1973].

An energy transfer rate of $(1.5-3.0) \mathrm{Wm}^{-2}$ is large enough to resupply the zonal mean kinetic energy of the layer (assuming $\bar{u}_{\mathrm{rms}} \approx 50 \mathrm{~m} \mathrm{~s}^{-1}$ ) in $2-4$ months, and is more than $10 \%$ of the emitted infrared heat flux from Jupiter, as was pointed out by Beebe et al. [1980]. On earth $\left\{K^{\prime} \bar{K}\right\}$ is also positive, but it is about $0.1 \%$ of the emitted infrared heat flux. The thermomechanical energy cycles must therefore be very different on Jupiter and the earth. One possibility is that the zonal flow $\bar{u}(y)$ extends much deeper than the eddies and therefore is affected on a time scale much longer than 4 months.

Acknowledgments. We thank members of the Voyager Project and the Image Processing Laboratory for their assistance. Brad Smith, as Imaging Science Team Leader, provided support and encouragement. Daniel Wenkert and Michael Kroupa helped with the data analysis. Contribution number 3489 of the Division of Geological and Planetary Sciences, California Institute of Technology, Pasadena, California 91125.

\section{REFERENCES}

Beebe, R. F., A. P. Ingersoll, G. E. Hunt, J. L. Mitchell, and J.-P. Müller, Measurements of wind vectors, eddy momentum transports, and energy conversions in Jupiter's atmosphere from Voyager 1 images, Geophys. Res. Lett., 7, 1-4, 1980.

Beebe, R. F., and L. A. Youngblood, Pre-Voyager velocities, accelera- 
tions and shrinkage rates of Jovian cloud features, Nature, 280 , 771-772, 1979.

Ingersoll, A. P., and J. N. Cuzzi, Dynamics of Jupiter's cloud bands, J. Atmos. Sci., 26, 981-985, 1969.

Ingersoll, A. P., R. F. Beebe, S. A. Collins, G. E. Hunt, J. L. Mitchell, J.-P. Müller, B. A. Smith, and R. J. Terrile, Zonal velocity and texture in the Jovian atmosphere inferred from Voyager images, $\mathrm{Na}$ ture, 280, 773-775, 1979.

Mitchell, J. L., R. F. Beebe, A. P. Ingersoll, and G. W. Garneau, Flow fields within Jupiter's Great Red Spot and white oval BC, $J$. Geophys. Res., this issue.

Oort, A. H., and J. P. Peixoto, The annual cycle of the energetics of the atmosphere on a planetary scale, J. Geophys. Res., 79, 27052719, 1974.

Rhines, P. B., Waves and turbulence on a beta-plane, J. Fluid Mech. $69,417-443,1975$.
Smith, B. A., and G. E. Hunt, Motions and morphology of clouds in the atmosphere of Jupiter, in Jupiter, edited by T. Gehrels, pp. 564585, University of Arizona Press, Tucson, 1976.

Weidenschilling, S. J., and J. S. Lewis, Atmospheric and cloud structure of the Jovian planets, Icarus, 20, 465-476, 1973.

Williams, G. P., Planetary circulations, 1, Barotropic representation of Jovian and terrestrial turbulence, J. Atmos. Sci., 35, 1399-1426, 1978.

Williams, G. P., Planetary circulations, 2, The Jovian quasi-geostrophic regime, J. Atmos. Sci, 36, 932-968, 1979.

(Received July 31, 1980;

revised December 1,1980 ;

accepted December 8, 1980.) 